\title{
Enhanced sandwich immunoassay using antibody-functionalized magnetic iron-oxide nanoparticles for extraction and detection of soluble transferrin receptor on a photonic crystal biosensor
}

Ross D. Peterson ${ }^{\mathrm{a}}$, Weili Chen ${ }^{\mathrm{b}}$, Brian T. Cunningham ${ }^{\mathrm{b}, \mathrm{c}}$, Juan E. Andrade ${ }^{\mathrm{a}, \mathrm{d} *}$

${ }^{a}$ Department of Food Science and Human Nutrition, University of Illinois at Urbana-Champaign, Urbana, IL 61801

${ }^{\mathrm{b}}$ Department of Electrical and Computer Engineering, University of Illinois at UrbanaChampaign, Urbana, IL 61801

${ }^{c}$ Department of Bioengineering, University of Illinois at Urbana-Champaign, Urbana, IL 61801

${ }^{\mathrm{d}}$ Division of Nutritional Sciences, University of Illinois at Urbana-Champaign, Urbana, IL 61801

*To Whom Correspondence should be addressed.

Address: 905 S Goodwin Ave. Urbana, IL 61801; E-mail: jandrade@illinois.edu

Abbreviations: BIND (Biomolecular Interaction Detection); fAb-IONs (functionalized iron-oxide nanoparticles with antibodies); IA (inverse sandwich assay); LoD (limit of detection); PC (photonic crystal); sTfR (soluble transferrin receptor); SA (standard sandwich assay). 


\begin{abstract}
Iron deficiency anemia (IDA) has detrimental effects on individuals and societies worldwide. A standard sandwich assay (SA) for the detection of soluble transferrin receptor (sTfR), a biomarker of IDA, on a photonic crystal (PC) biosensor was established, but it was susceptible to non-specific signals from complex matrixes. In this study, iron-oxide nanoparticles (fAb-IONs) were used as magnetic immuno-probes to bind sTfR and minimize non-specific signals, while enhancing detection on the PC biosensor. This inverse sandwich assay (IA) method completely bound sTfR with low variability $(<4 \%$ RSD) in buffer and allowed for its accurate and precise detection in sera (Liquichek ${ }^{\mathrm{TM}}$ control sera) on the PC biosensor using two certified ELISAs as reference methods. A linear dose-response curve was elicited at the fAb-IONs concentration in which the theoretical binding ratio (sTfR:fAb-IONs) was calculated to be $<1$ on the IA. The LoDs for sTfR in the SA and IA were similar $(\mathrm{P}>0.05)$ at 14 and $21 \mu \mathrm{g} / \mathrm{mL}$, respectively. The inherent imprecision of the IA and reference ELISAs was $\sigma(\delta)=0.45 \mu \mathrm{g} / \mathrm{mL}$ and the mean biases for Liquichek ${ }^{\mathrm{TM}} 1,2$ and 3 were $0.18,0.19$ and $-0.04 \mu \mathrm{g} / \mathrm{mL}$, respectively. Whereas the inherent imprecision of the SA and reference ELISAs was $\sigma(\delta)=0.52 \mu \mathrm{g} / \mathrm{mL}$ and the biases for Liquichek $^{\mathrm{TM}} 1,2$ and 3 were $0.66,0.14$ and $-0.67 \mu \mathrm{g} / \mathrm{mL}$, respectively. Thus, unlike the SA, the IA method measures sTfR with the same bias as the reference ELISAs. Combined magnetic separation and detection of nutrition biomarkers on PC biosensors represents a facile method for their accurate and reliable quantification in complex matrixes.
\end{abstract}

Keywords: biosensor; magnetic separation; iron deficiency; iron-oxide nanoparticles; photonic crystal; soluble transferrin receptor 


\section{Introduction}

Iron deficiency is estimated to account for half of the two billion anemia cases worldwide (WHO, 2008). When untreated, iron deficiency anemia (IDA) has shown to negatively affect cognition in children (Sachdev et al., 2005), productivity in adults (Horton and Ross, 2003) and pregnancy in women (Black et al., 2008). In addition to hemoglobin, two protein biomarkers commonly used to diagnose IDA are ferritin and soluble transferrin receptor (sTfR). Although ferritin is used by the World Health Organization (WHO) as a biomarker of iron deficiency, its accumulation in the body's acute-phase response during episodes of infection or inflammation (Worwood, 1979) limits its diagnostic value (WHO, 2008). sTfR, however, is unaffected by infection and increases when red blood cell production is upregulated (Ward, 1987). Although sTfR has diagnostic utility when combined with ferritin, it was not until 2010 (Thorpe et al., 2010) when the measurement of sTfR for diagnosis was standardized. As a result of an international collaborative effort, the WHO developed a reference standard reagent (07/202) to help standardize sTfR immunoassays.

Aside from hemoglobin determination methods, current methodologies to measure IDA biomarkers require specialized equipment, trained technicians, and medical laboratories for analysis (Erhardt et al., 2004; Pfeiffer et al., 2007). As a consequence, large-scale surveying studies are not feasible to identify people suffering from or at risk for IDA in rural areas of lowincome countries. This hinders the ability of nutrition and health interventions to assist those most in need. Thus, diagnostic platforms amenable for use in field settings and that provide quick and actionable results are critically needed to facilitate widespread diagnosis of nutrient deficiencies and support development programs. 
Photonic crystal (PC) biosensors have shown potential as a prospective diagnostic platform to measure biomarkers of IDA at the point of care (Gallegos et al., 2013). PCs are periodic subwavelength grating structures that reflect a narrow band of wavelengths when illuminated by a broadband light source (Cunningham et al., 2002) at the resonance conditions. Attachment of biomolecules (e.g. proteins) upon the near field ( $200 \mathrm{~nm})$ of the biosensor surface increase the refractive index (Arakawa and Kita, 1999) of the resonance mode and cause a shift of peak wavelength value (PWV) to a higher value; thus, representing a simple mechanism for biomarker detection.

<<Insert Figure 1 and Caption here〉>

PC biosensor detection systems have various diagnostic and screening applications in DNA microarrays (Mathis et al., 2010), cancer cell analysis (Chen et al., 2013), virus detection (Shafiee et al., 2014) protein detection (Peterson et al., 2014a) and high throughput drug screening (Heeres and Hergenrother, 2002). Although current PC biosensor methodologies require a laboratory setting, a portable optical interface capable of converting a smartphone into a detection instrument was recently designed for the PC biosensor (Gallegos et al., 2013). Nevertheless, the most significant challenge continues to be the accurate and sensitive detection of biomarkers in complex matrixes (Marks, 2002; Kyprianou et al., 2013).

Complex matrixes (i.e. serum and whole blood) containing variable amounts of interfering proteins are the primary cause of non-specific binding and poor signal-to-noise responses (Kyprianou et al., 2013), which results in unreliable data. These can lead to false-negative or false-positive clinical results that instead increase healthcare costs and burden the health of 
patients. IDA misdiagnoses can have harmful side effects on patients, especially when providing iron supplementation unnecessarily to children with malaria (Sazawal et al., 2006). Thus, in the development of point-of-care tools to assess patients' nutritional status in low-income field settings, samples must be analyzed using field-friendly protocols that do not jeopardize diagnostic reliability.

Previously, the authors established the use of PC biosensors as a standard sandwich assay (SA) to detect and quantify ferritin as a potential platform for iron deficiency assessment (Fig. 1A), where the signal from bound ferritin was further amplified by the use of antibodies conjugated to colloidal iron-oxide nanoparticles (IONs) (Peterson et al., 2014a). In addition to ferritin, detection of sTfR in a dose-response manner was verified. However, in an attempt to measure the WHO reference standard of sTfR in a solution of $100 \mathrm{mg} / \mathrm{mL}$ of bovine serum albumin, the PC biosensor overestimated the expected sTfR concentration $2 \mathrm{x}$ compared to the same sTfR concentration in buffer (Peterson et al., 2014b). Magnetic nanoparticles have recently been used to isolate biomolecules of interest from complex matrixes and to enhance sensitivity by concentrating the biomarker of interest on a surface plasmon resonance biosensor (Soelberg et al., 2009). However, the ability to reduce interference signals in complex matrixes (i.e. serum) using a PC biosensor and provide method validation information on inaccuracy and imprecision as part of a combined assay to extract and measure protein biomarkers has not been studied.

In the present work, the development of an inverse sandwich assay (IA) (Fig. 1B) is described where antibody-conjugated IONs are used as magnetic immuno-probes to decrease interference signals for the accurate and reliable determination of sTfR in serum.

\section{Materials and Methods}




\subsection{Reagents.}

3-glycidoxy-propyl-trimethoxy-silane (GTPMS), and $\mathrm{NaOH}$ were purchased from SigmaAldrich; phosphate buffer saline (PBS), zeba spin columns 40K $0.5 \mathrm{~mL}$ and StartingBlock blocking buffer from Pierce (Thermo Fisher Scientific). Double deionized water (DDW) was used in all experiments. ELISA kits, antigen (sTfR) and antibodies (Ab). Human sTfR ELISA kits were purchased from BioVendor (RD194011100) and R\&D Systems (DTFR1). Liquichek ${ }^{\mathrm{TM}}$ serum controls (Bio-Rad Laboratories, Inc. Lot \#52480) were diluted in PBS to measure inaccuracy and imprecision in replication experiments and comparison of methods experiments. The monoclonal mouse anti-human sTfR was used as the capture antibody in the PC biosensor assay and was purchased from Abcam ${ }^{\circledR}$ (ab38168). A monoclonal mouse anti-human sTfR antibody was the detection $\mathrm{Ab}$ in the PC biosensor and was purchased from Abcam® $(\mathrm{ab} 10249)$. Iron-oxide nanoparticle conjugation kit was purchased from Ocean NanoTech (ICK-30-005).

\subsection{PC biosensor and readout system.}

Readers are referred to Cunningham et al., 2002; Peterson et al., 2014a; Cunningham et al., 2004) where there is detailed information about PC biosensor and readout system. PC microplates (384-well) were purchased from SRU Biosystems, Inc. The Biomolecular Interaction Detection system (BIND; SRU Biosystems, Inc.) was used to measure biomolecule interaction on biosensor surface (i.e. antibody interactions with antigen). The detection instrument illuminates the PC with a broadband light source ( $\lambda$ range $400-700 \mathrm{~nm}$ ) that provides collimated light at normal incidence via an optical fiber positioned below the biosensor microplate. The system contains 8-parallel readout heads, and is capable of measuring the Peak Wavelength Value (PWV) of all wells in a 384-well microplate in $\sim 10$ seconds. For all the experiments 
presented using the PC biosensor, the quantities are reported as PWV shifts in units of wavelength (nm).

\subsection{Transmission Electron Microscope}

Philips CM200 transmission electron microscope (TEM) was used to take images of IONs. The in-house procedure revised in 2006 by Jakstys and Klintsova was followed to take TEM images TEM (Jakstys and Klintsova, 2006). Images were taken of functionalized IONs with antibody (fAb-IONs), sTfR-fAb-IONs complexes before magnetic separation and sTfRfAb-IONs complexes after magnetic separation.

\subsection{Preparation of PC biosensor.}

\subsubsection{Epoxy-silanization of PC biosensor surface.}

PC biosensor surface was functionalized similarly as previously described. ${ }^{16}$ Briefly, each well was incubated for $1 \mathrm{~h}$ at $23^{\circ} \mathrm{C}$ in a solution of $0.1 \mathrm{M} \mathrm{NaOH}$, before sonication (Fisher Scientific Isotemp202 Heater Ultrasonic bath) for $15 \mathrm{~min}$. After aspiration and blotting, wells received $15 \mu \mathrm{L}$ of $2.5 \%$ GTPMS and $10 \mathrm{mM}$ acetic acid in ethanol solution and were left to incubate for $1 \mathrm{~h}$ at $23^{\circ} \mathrm{C}$. Finally, wells were aspirated and washed twice with ethanol and dried under nitrogen stream before they were ready to be assayed.

\subsubsection{Capture monoclonal Antibody immobilization.}

An aliquot of $15 \mu \mathrm{L}$ at $40 \mu \mathrm{g} / \mathrm{mL}$ of capture anti-sTfR antibodies was dispensed into all epoxy-silanized wells. The PC microplate was sealed with tape (Pierce, Thermo Fisher Scientific) and left at $23^{\circ} \mathrm{C}$ for $5 \mathrm{~h}$. Then, wells were washed with PBS three times. The PWV 
corresponding to capture antibody immobilized on sensor surface was measured relative to baseline.

\subsubsection{Blocking step.}

Next, an aliquot of $20 \mu \mathrm{L}$ undiluted StartingBlock was pipetted into all active and negative control wells. PC microplates were incubated for $45 \mathrm{~min}$ at room temperature and then washed with PBS three times. The PWV shift was measured relative to baseline. At this step, the PC sensor is ready to use in the SA to bind sTfR, or in the IA to bind complexes of fAb-IONs and sTfR.

\subsection{Preparation of functionalized iron-oxide nanoparticles (fAb-IONs).}

Detection antibodies were functionalized (fAb) to iron-oxide nanoparticles (30 $\mathrm{nm})$ by the vendor (Ocean NanoTech, LLC) and described in a previous study without modifications

(Peterson et al., 2014a; Xu et al., 2011). Conjugation was verified by gel electrophoresis from Ocean NanoTech (data not shown). Conjugations are expressed as mg of Fe per mL. TEM images were taken after fAb-IONs functionalization.

\section{6. sTfR detection using standard sandwich assay.}

In this assay the antigen was added and incubated with the capture antibody in the PC biosensor first, and then, the fAb-IONs were added to create the sandwich (Fig. 1A). For this, five concentrations $(0.01-0.2 \mu \mathrm{g} / \mathrm{mL})$ of sTfR $(15 \mu \mathrm{L})$ were directly applied to the PC biosensor and their respective PWV was measured relative to the PWV of the previous blocking step to develop a standard curve. After $20 \mathrm{~min}, 15 \mu \mathrm{L}$ of fAb-IONs at a concentration of $0.0241 \mathrm{mg}$ 
$\mathrm{Fe} / \mathrm{mL}$ were added to each well and data on binding kinetics were collected for 20 hours, which allowed each well to reach steady-state binding conditions.

\section{7. sTfR detection using inverse sandwich assay.}

In the IA, the antigen-containing sample and fAb-IONs were mixed first in a different tube, then after magnetic separation, removal of supernatant and resuspension in buffer, the complex was added and incubated with the capture antibody on the PC biosensor (Fig. 1B). The mixture of fAb-IONs and sTfR was incubated at room temperature $\left(23^{\circ} \mathrm{C}\right)$ on a shaker $(400 \mathrm{rpm})$ for $1 \mathrm{~h}$ before magnetic separation was applied using the SuperMag Multitube Separator ${ }^{\mathrm{TM}}$ (Ocean NanoTech) for $1 \mathrm{~h}$ in $1.5 \mathrm{~mL}$ micro-centrifuge tubes. Specific reconstitution and concentration information of fAb-IONs and sTfR for each experiment are described below. TEM images were taken before and after magnetic separation to compare aggregation.

\subsection{Optimization of fAb-ION concentration for removal of sTfR and binding ratio determination.}

fAb-IONs were serially diluted in BioVendor dilution buffer to final concentrations of $0.0625,0.03125$, and $0.015625 \mathrm{mg} \mathrm{Fe} / \mathrm{mL}$. These concentrations were chosen based on previous binding data from the SA in which a linear dose-response curve was established using $0.0241 \mathrm{mg}$ $\mathrm{Fe} / \mathrm{mL}$. BioVendor sTfR standards were diluted to final concentrations of $0.2,0.3,0.4$ and 0.5 $\mu \mathrm{g} / \mathrm{mL}$ and assayed with each of the three aforementioned fAb-IONs concentrations. The highest sTfR concentration $(0.5 \mu \mathrm{g} / \mathrm{mL})$ was chosen because it is near the cut-off level of sTfR for iron deficiency (Mei et al., 2012; Phiri et al., 2009) in serum when diluted 1:10. This is also the upper limit of the IA linear range. During the magnetic separation of each treatment, after the fAb- 
IONs pellet was attracted to the magnetic side of the tube and the solution was transparent, the supernatant was removed. Then, it was diluted 2.5 times before sTfR analysis with the BioVendor ELISA. Diluting 2.5 times was necessary to reach sufficient assaying volume for the ELISA. The sTfR amount in the supernatant was used to calculate extraction recovery using the fAb-IONs. Binding ratios were calculated in terms of sTfR:fAb-IONs molecules. The molar concentration of fAb-IONs was provided by Ocean NanoTech and the molecules of sTfR were calculated by using the molecular weight ( $85 \mathrm{kDa}$ ) of sTfR (Speeckaert et al., 2010).

\subsection{Determination of inaccuracy and bias in the analysis of sTfR from Liquichek ${ }^{\mathrm{TM}}$ control} sera.

To quantify sTfR in three Liquichek ${ }^{\mathrm{TM}}$ control sera, the fAb-IONs were diluted to a final concentration of $0.25 \mathrm{mg} \mathrm{Fe} / \mathrm{mL}$ before incubation with all samples on the IA. The SA used a final concentration of fAb-IONs at $0.0241 \mathrm{mg} \mathrm{Fe} / \mathrm{mL}$ to measure all samples. All other parameters described below remained constant for both the IA and SA on the PC biosensor. BioVendor standard concentrations $(0.01-0.5 \mu \mathrm{g} / \mathrm{mL})$ were used to develop the standard curve on the PC biosensor and determine actual concentrations of sTfR in Liquichek ${ }^{\mathrm{TM}}$ control sera. Liquichek $^{\mathrm{TM}}$ control sera were diluted 25 times to total protein concentrations of approximately 2.0, 3.0, $4.0 \mathrm{mg} / \mathrm{mL}$ for Liquichek ${ }^{\mathrm{TM}} 1,2$ and 3, respectively. These protein concentrations are based on data provided by Bio-Rad Laboratories, Inc. Dilutions at 1:25 were necessary to bring sTfR concentration in Liquichek ${ }^{\mathrm{TM}}$ sera to within the linear range of both assay platforms. Two commercial ELISAs were used to determine the reference concentrations of sTfR in the Liquichek $^{\mathrm{TM}}$ sera. During magnetic extraction in the IA the supernatant was removed and discarded, and the pellet containing sTfR complexed to fAb-ION was resuspended in PBS to 
original volume before analysis on the PC biosensor. Inaccuracy and bias from measuring the Liquichek $^{\mathrm{TM}}$ sera was quantified using difference plots (Petersen et al., 1997) for both PC biosensor assay types. These parameters were determined by comparing the IA and SA of the PC biosensor to the average sTfR concentration measured from the two reference ELISA methods. The inherent imprecision of the test methods was calculated following a previously published equation (Petersen et al., 1997):

$$
\sigma^{2}(\delta)=\sigma_{T}^{2}+\sigma_{R}^{2}
$$

where $\left[\sigma_{T}^{2}\right]$ is the variance of either test method (i.e. IA and SA), $\left[\sigma_{R}^{2}\right]$ is the variance of the reference methods (i.e. ELISAs) and $\left[\sigma^{2}(\delta)\right]$ is the total inherent imprecision of the test and reference methods. Briefly, constant analytical standard deviations are presumed, and thus it is equal to $\sigma_{T}+\sigma_{R}$. When the two methods are identical, it is expected that $68 \%$ of differences will be distributed around 0 between $0 \pm 1 \sigma(\delta)$ and $95 \%$ of differences will be between $0 \pm 2 \sigma(\delta)$.

\subsection{Determination of precision and limit of detection of $\mathrm{PC}$ platform and commercial ELISAs.}

Imprecision was determined on each platform by measuring Liquichek ${ }^{\mathrm{TM}}$ control serum 2 in replication experiments. Intra-assay imprecision was measured using triplicates for each experiment. Inter-assay imprecision was calculated using data from two experiments with either duplicates or triplicates. Limit of detection (LoD) was also determined as described previously (Armbruster and Pry, 2008). 


\section{Results and Discussion}

\subsection{Determination of fAb-IONs concentration to achieve maximum sTfR removal and}

binding ratios on inverse sandwich assay.

A $4 \times 3$ factorial design experiment was used to evaluate the ability of fAb-IONs in binding sTfR in buffer, where four concentrations of sTfR were paired with three concentrations of fAb-IONs. After evaluation of supernatants using ELISA, the highest concentration of fAb-IONs $(0.0625$ $\mathrm{mg} \mathrm{Fe} / \mathrm{mL}$ ) recovered $>89 \%$ of all the sTfR levels (Fig. 2).

$<<$ Insert Figure 2 and Caption here >>

The lowest concentration of fAb-IONs $(0.0156 \mathrm{mg} \mathrm{Fe} / \mathrm{mL})$, however, only recovered $50 \%$ of the highest concentration of sTfR $(0.5 \mu \mathrm{g} / \mathrm{mL})$. All these data points had a relative standard deviation (RSD) of less than $4 \%$. It was expected that a higher concentration of fAb-IONs would potentially remove nearly $100 \%$ of sTfR from solution. The high sTfR recoveries at 0.0625 and $0.03125 \mathrm{mg} \mathrm{Fe} / \mathrm{mL}$ supported the experimental data observed from the SA on the PC biosensor, where a linear dose-response to sTfR was created using 0.03125 (not shown) and $0.0625 \mathrm{mg}$ Fe/mL fAb-IONs (Fig. 3A, - •-).

$<<$ Insert Figure 3 and Caption here>>

Thus, fAb-IONs at $0.0625 \mathrm{mg} \mathrm{Fe} / \mathrm{mL}$ were used to magnetically extract sTfR standards in the IA. Building on the previous data shown in Fig. 2, the binding ratios of sTfR:fAb-IONs for each concentration of fAb-IONs and sTfR in the 4x3 factorial design were calculated. The binding ratios ranged from 1:1-2:1, 2:1-4:1, and 3:1-5:1 at $0.0625 \mathrm{mg} \mathrm{Fe} / \mathrm{mL}, 0.03125 \mathrm{mg} \mathrm{Fe} / \mathrm{mL}$ and 
$0.01562 \mathrm{mg} \mathrm{Fe} / \mathrm{mL}$, respectively, for each of the four concentrations of sTfR. The significance of these binding ratios will be discussed in the next section.

\subsection{Optimum concentration of fAb-IONs to develop a dose-response curve measuring sTfR using the PC biosensor.}

Based on the experimental data described in Fig. 2, it was hypothesized that by using the highest concentration of fAb-IONs applied in the recovery experiments (i.e. $0.0625 \mathrm{mg} \mathrm{Fe} / \mathrm{mL}$ ) to magnetically extract a range of increasing concentrations of sTfR standards in the IA would lead to a similar linear dose-response as obtained in the SA. However, when three concentrations of sTfR (i.e. $0.05,0.1$ and $0.2 \mu \mathrm{g} / \mathrm{mL}$ ) were assayed using $0.0625 \mathrm{mg} \mathrm{Fe} / \mathrm{mL}$ of fAb-IONs in the IA, no linear dose-response curve was obtained (Fig. 3A,-- -). Thus, to optimize the concentration of fAb-IONs necessary to produce a linear dose-response curve in the IA, one concentration of sTfR (i.e. $0.1 \mu \mathrm{g} / \mathrm{mL}$ ) was assayed with six serially diluted concentrations of fAb-IONs (0.024 - $0.5 \mathrm{mg} \mathrm{Fe} / \mathrm{mL})$. Fig. 3B shows the binding characteristics of sTfR in the IA, where increased concentrations of fAb-IONS elicited higher PWV responses. Responses did not follow a linear pattern. At the lower end, from 0.024 to $0.1 \mathrm{mg} \mathrm{Fe} / \mathrm{mL}$, PWV increments were steady but low. At higher fAb-IONS concentrations, increments in PWV were larger. The evaluation of $0.25 \mathrm{mg} \mathrm{Fe} / \mathrm{mL}$ fAb-IONs in the IA resulted in a linear dose-response curve (Fig. 3A,- $\square$-). Therefore, although $0.0625 \mathrm{mg} \mathrm{Fe} / \mathrm{mL}$ fAb-IONs removed $>89 \%$ of several sTfR concentrations $(0.1-0.5 \mu \mathrm{g} / \mathrm{mL})$, this was not optimal for development of a linear dose-response curve, and thus, a higher concentration of fAb-IONs was needed.

These results, although perplexing, can be explained based on the nature of the fAb-IONs and the differential binding of sTfR in either assay type. In the SA, the antigens are added $20 \mathrm{~min}$ 
before the fAb-IONs, which allows time to interact with the capture antibodies immobilized on the sensor surface. As a result, the antigen reaches steady-state binding equilibrium with the capture antibody before the fAb-IONs are added for detection amplification. This configuration enables maximum binding efficiency between the capture antibody and antigens as well as the fAb-IONs and antigens. As a result of this configuration using the SA, despite the difference in fAb-IONs concentrations, a maximum PWV response was reached when measuring the same sTfR concentration albeit with different binding kinetics (Fig. 3C). In the IA, however, the antigen are first bound to fAb-IONs forming complexes, and then, magnetically extracted as a whole unit. These sTfR:fAb-ION networks formed due to the aggregation interactions continue even after re-suspension and detection on the PC biosensor. Early complexation between sTfRfAb-IONs prevented sTfR from binding with the capture antibodies and eliciting a maximum response similar to that observed in the SA prior to adding fAb-IONs (Fig. 3C). Nonetheless, as the concentration of fAb-IONs increased, and the ratio of sTfR:fAb-IONs decreased, the response approached the maximum response observed in the SA. At the fAb-IONs concentration where a linear dose-response curve was elicited (i.e. $0.25 \mathrm{mg} \mathrm{Fe} / \mathrm{mL}$ ), the theoretical binding ratio was calculated to be $<1$ (less sTfR molecules per fAb-ION), which was in contrast to the lower fAb-ION concentrations shown in the previous section (i.e. $0.0625,0.03125$ and 0.01562 $\mathrm{mg} \mathrm{Fe} / \mathrm{mL}$ ) where the binding ratios were $>1$ (more sTfR molecules per fAb-ION).

Although this binding mechanism is not completely understood, these data suggest a binding ratio $<1$ leads to a lower amount of sTfR:fAb-ION network formation due to the lesser amount of sTfR per fAb-ION, and thereby, promotes interactions between sTfR and the capture antibody more similar to the SA. TEM images taken after magnetic separation of the sTfR-fAb-IONs complexes and prior to assaying on the PC biosensor did not show more aggregation compared 
to TEM images of the fAb-IONs alone and sTfR-fAb-IONs complexes before magnetic separation (Fig. 4). This lower amount of fAb-ION aggregation is considering that the 30-nm IONs are nearly superparamagnetic and carry a single magnetic domain (Lu et al., 2007). Conversely, at a ratio $>1$, there is more sTfR per fAb-ION, which is thought to lead to more sTfR:fAb-ION network formation between the sTfR and fAb-IONs, and thus, inhibit the sTfR from interacting with the capture antibodies on the biosensor surface. Therefore, although a lower concentration of fAb-IONs extracted $>89 \%$ of sTfR through magnetic separation, a high proportion of sTfR remained "hidden" and unable to interact with the capture antibodies. Similar aggregation or network formations were observed when biotinylated IONs interacted with streptavidin in situ under a magnetic field within an SPR biosensor detection system (Lee et al., 2012). Despite these explanations, more studies are needed to examine the exact mechanism causing differential responses observed at $0.25 \mathrm{mg} \mathrm{Fe} / \mathrm{mL}$ and those concentrations at and below $0.06125 \mathrm{mg} \mathrm{Fe} / \mathrm{mL}$.

$<<$ Insert Figure 4 and Caption here>>

\subsection{Standard curve and kinetic binding curve of inverse sandwich assay on PC biosensor.}

A linear dose-response curve was obtained with $0.25 \mathrm{mg} \mathrm{Fe} / \mathrm{mL}$ of fAb-IONs in the IA (Fig. 4A).

$<<$ Insert Figure 5 and Caption here >>

The linear curve represents five concentrations of sTfR, $0.02-0.5 \mu \mathrm{g} / \mathrm{mL}$. Within this range, the sTfR dose-responses were linear, with an $\mathrm{R}^{2}$ value of 0.998 . The increased PWV kinetic 
responses as a result of binding with sTfR-fAb-IONs complexes can be explained by the nature of the PC detection system. The PC biosensor is a periodic subwavelength grating structure that can detect the existence of the attached complexes under optical resonance (Chen et al., 2014). The evanescent electric field generated by the resonance has an exponential decaying intensity versus the vertical distance above PC surface, as shown by the following equation:

$$
E(z)=E(0)^{-z / d}
$$

where $E(z)$ represents the electric field intensity at a certain distance $z$ above the PC surface, $E(0)$ is the intensity at the surface interface, and $d$ is called the penetration depth, which is also the effective detection range of the PC biosensor (Chen et al., 2014). Eq. 2 indicates that the interaction between the sTfR-fAb-IONs complexes and the PC biosensor is highly dependent on their distance from the surface. In the IA, at $0.25 \mathrm{mg} \mathrm{Fe} / \mathrm{mL}$ of fAb-IONs, the addition of the sTfR-fAb-IONs complexes in one step allows the complexes to interact with the immobilized capture antibodies and arrange themselves close to the PC biosensor surface, where the electric field is maximized, and thus, producing a strong detection signal. Based on the experimental data, the higher the fAb-IONs concentration in the IA, the greater the response will be as it approaches the maximum steady-state equilibrium of the SA (Fig. 3C). As described in the previous section, however, unless there is a $<1$ ratio of sTfR to fAb-IONs to amplify the signal, which was determined to be above $0.1 \mathrm{mg} \mathrm{Fe} / \mathrm{mL}$, no dose-response could be achieved (Fig. 3A and $\mathrm{B})$.

The ability to track kinetic binding on the PC biosensor allows for the development of realtime dynamic binding curves. Fig. 5B shows the dynamic binding curves for three sTfR concentrations at one concentration of fAb-IONs (i.e. $0.25 \mathrm{mg} \mathrm{Fe} / \mathrm{mL}$ ) in the IA. Responses in this assay reached equilibrium by $200 \mathrm{~min}$; however, differences among concentrations are 
significant even within $30 \mathrm{~min}$. As described in Eq. 2, the mechanism for the kinetic binding of the antigen-fAb-IONs complexes involves the mass of these complexes displacing volume in the detectable area of the PC biosensor. Due to the multivalency of the fAb-IONs during magnetic separation, each fAb-ION can bind numerous sTfR (Yang et al., 2008; Saha et al., 2014). The antigen-fAb-IONs complexes with large cross-sections migrated towards the PC biosensor surface at a high velocity, and therefore reaching equilibrium within a short time.

\subsection{Inaccuracy of inverse and standard assays on the PC biosensor in measuring sTfR from reference materials.}

The interference/non-specific binding in the measurement of sTfR in a complex matrix (i.e. diluted sera) was evaluated with both assays on the PC biosensor. These experiments showed that the IA was able to extract, detect and quantify sTfR in Liquichek ${ }^{\mathrm{TM}}$ sera 1,2 , and 3 with minimal inaccuracy (i.e. bias) compared to the two reference ELISA methods. The average bias for Liquichek ${ }^{\mathrm{TM}} 1,2$ and 3 was $0.18,0.19$ and $-0.04 \mu \mathrm{g} / \mathrm{mL}$, respectively. This means the IA slightly overestimated the sTfR in two of three Liquichek ${ }^{\mathrm{TM}}$ samples compared to the mean of the reference ELISAs. The inherent imprecision of the IA and reference ELISAs was calculated as $\sigma(\delta)=0.45 \mu \mathrm{g} / \mathrm{mL}$. These data indicated that $68 \%$ of the differences between methods should be between 0 and $0.45 \mu \mathrm{g} / \mathrm{mL}$ and $95 \%$ of differences between 0 and $0.90 \mu \mathrm{g} / \mathrm{mL}$ of sTfR. Although the sample size was small, $83 \%$ of points fell within $0 \pm 0.45 \mu \mathrm{g} / \mathrm{mL}$ and $100 \%$ of points fell between $0 \pm 0.90 \mu \mathrm{g} / \mathrm{mL}$ (Fig. 6A). 
Therefore, due to a small bias and inherent imprecision that fits the normal distribution, the IA method does not perform statistically different from the reference ELISA tests.

The SA, however, had a greater bias measuring sTfR in Liquichek ${ }^{\mathrm{TM}}$ sera when compared to the reference ELISA methods. As shown in Fig. 6B, the biases for Liquichek ${ }^{\mathrm{TM}} 1,2$ and 3 were $0.66,0.14$ and $-0.67 \mu \mathrm{g} / \mathrm{mL}$, respectively. Consequently, the SA overestimated (positive bias) the sTfR concentration in Liquichek ${ }^{\mathrm{TM}} 1$ and 2 and underestimated (negative bias) Liquichek ${ }^{\mathrm{TM}} 3$. The inherent imprecision of the SA and reference ELISAs was calculated as $\sigma(\delta)=0.52 \mu \mathrm{g} / \mathrm{mL}$. As described above, the inherent bias indicates that $68 \%$ and $95 \%$ of the differences should fall between $0 \pm 0.52$ and $0 \pm 1.04 \mu \mathrm{g} / \mathrm{mL}$. Although data from Liquichek ${ }^{\mathrm{TM}} 2$ followed this distribution, data from Liquichek ${ }^{\mathrm{TM}} 1$ and 3 had $80 \%$ of the replicates with a difference greater than $0.52 \mu \mathrm{g} / \mathrm{mL}$. Thus, the SA performed statistically different from the reference ELISA tests in measuring sTfR. The bias observed indicated that these samples contained some non-specific component, more likely serum proteins.

\subsection{Imprecision and limit of detection (LoD) of inverse assay, standard assay, and commercial ELISAs.}

The imprecision of the detection platforms were compared to each other through intra- and inter-assay replication studies, measuring sTfR in Liquichek ${ }^{\mathrm{TM}} 2$ serum (Table 1).

$<<$ Insert Table 1 and Caption here >

These data indicated the intra-assay imprecision of the SA and IA on PC biosensor was higher than the commercial ELISA assays measuring sTfR from the reference sample. Despite this, 
based on the inaccuracy and inherent imprecision shown in the difference plots (Fig. 6), the IA performs similar to both ELISA tests in the quantification sTfR. To put the imprecision of the IA and SA in clinical perspective, based on biological variation data (Bailey et al., 2014), the maximum allowable random error (i.e. standard deviation) for sTfR is $0.23 \mu \mathrm{g} / \mathrm{mL}$ for intraassay and $0.30 \mu \mathrm{g} / \mathrm{mL}$ for inter-assay at a sTfR cut-off concentration of $5.3 \mu \mathrm{g} / \mathrm{mL}$. Experimentally the SDs of the SA for the intra- and inter-assay replication studies were 0.19 and $0.13 \mu \mathrm{g} / \mathrm{mL}$, respectively. Likewise, in the IA, these were 0.12 and $0.23 \mu \mathrm{g} / \mathrm{mL}$, respectively. Therefore, for both assays the SDs were within the total allowable error for sTfR, indicating that the random error observed has no clinical significance when measuring sTfR on the PC biosensor. The inter-assay imprecision of the SA was small due to interference that falsely homogenized the responses. The LoD of the IA and SA were lower than those from the reference ELISAs (Table 1). Due to the IONs amplification in the PC biosensor assays, the LoD on this platform is 5-fold to 40-fold lower than the commercial ELISAs. The low LoD of the PC biosensor is particularly useful when measuring small quantities of biomolecules in blood. In the case of sTfR, the low LoD is not as beneficial because in an iron-deficient individual, normal circulating sTfR concentrations increase above the cut off of $5.3 \mu \mathrm{g} / \mathrm{mL}$. Therefore, the upper detection limit of the linear range (i.e. $0.5 \mu \mathrm{g} / \mathrm{mL}$ ) is more important to evaluate the utility of this assay when assessing sTfR status. Regarding the relevance of this upper detection limit in the IA, a simple 1:10 sample dilution would be needed to determine whether the individual is above or below the cutoff; in which a value greater than $0.5 \mu \mathrm{g} / \mathrm{mL}$ after dilution would indicate deficiency, whereas a value less than $0.5 \mu \mathrm{g} / \mathrm{mL}$ would indicate sufficiency. Accurate diagnosis of IDA requires the use of at least two biomarkers, such as ferritin and sTfR. Unlike sTfR, ferritin levels can fall below $30 \mathrm{ng} / \mathrm{mL}$ in IDA individuals. Thus, a low LoD achieved through 
the IA method could help isolate, detect and measure this biomarker as well as we have shown previously (Peterson et al., 2014a).

\subsection{Limitations.}

Despite these encouraging data, there are areas of further research necessary to continue the technological process development. The optimization of functional concentrations of several assay components is required to maximize its performance. It is known that EDC-NHS functionalization used in this study to immobilize antibodies to IONs results in random orientation. Future studies will test Protein A functionalization or streptavidin functionalization to improve antibody orientation on the IONs that could potentially improve antigen detection (Soelberg et al., 2009). Furthermore, these studies require confirmation using sera from a larger sample of subjects representing populations with normal and deficient levels of sTfR. In terms of point-of-care amenability, more studies are needed to test the smartphone platform developed for the PC biosensor (Gallegos et al., 2013), and test microfluidic concepts that may improve the extraction of biomarkers from biological samples. Regarding assay time, future optimization studies are needed to determine the optimal time need for antigen-fAb-IONs binding before magnetic separation as well the optimal time needed for magnetic separation.

\section{Conclusion}

A detection protocol (inverse sandwich assay) that combines magnetic separation of a protein biomarker of iron deficiency with immuno-probes and their detection using a PC biosensor was established. The IA was able to extract sTfR from buffer and serum, elicit a linear standard curve, and perform similarly to two certified ELISAs in terms of precision and bias in the quantification of sTfR from three control sera. This agreement in quantifying sTfR in sera suggests the IA assay removes the interfering compounds that cause non-specific binding in the 
SA. Although, the SA was capable of reaching higher sTfR responses on the PC biosensor from lower concentrations of fAb-IONS, it tended to overestimate (positive bias) sTfR at low concentrations and underestimate (negative bias) at high concentrations, reducing its diagnostic potential. While further optimization is needed, the experimental protocol presented herein provides a simple process whereby biomarkers are magnetically separated from a complex matrix to remove non-specific binding signals; a problem often associated with biosensors' performance at the point of care. Future studies will examine how pre-concentration of samples after magnetic separation can improve sensitivity, and ultimately, the usefulness of this assay when measuring biomarkers at low physiological concentrations.

\section{Acknowledgments}

This project was supported by USDA Hatch project ILLU-971-342 (J.E.A., B.T.C.); American Society for Nutrition Pfizer Predoctoral Fellowship (R.D.P.).

R.D.P. and J.E.A. designed research; R.D.P. conducted research; R.D.P. and W.C. analyzed data; R.D.P., W.C., B.T.C. and J.E.A. contributed to writing of the paper. J.E.A. had primary responsibility for final content. All authors read and approved the final manuscript.

\section{References}


- Arakawa, T., Kita, Y., 1999. Anal. Biochem. 271, 119-120.

- Armbruster, D., Pry, T., 2008. Clin. Biochemist Rev. 29 (Suppl. 1), S49-S52.

- Bailey, D., Bevilacqua, V., Colantonio, D., Pasic, M., Perumal, N., Chan, M., Adeli, K., 2014. Clin. Chem. 60, 518-529.

- Black, R., Allen, L., Bhutta, Z., Caulfield, L., de Onis, M., Ezzati, M., Mathers, C., Rivera, J., 2008. Lancet. 371, 243-260.

- Chen, W., Long, K., Lu, M., Chaudhery, V., Yu, H., Choi, J.S., Polans, J., Zhuo, Y., Harley, B.A., Cunningham, B.T., 2013. Analyst. 138.20, 5886-5894. Analyst. 138.20 (2013): 5886-5894.

- Chen, W., Long, K., Yu, H., Tan, Y., Choi, J., Harley, B., Cunningham, B., 2014. Analyst. 139, 5954-5963.

- Cunningham, B., Li, P., Lin, B., Pepper, J., 2002. Sens. Actuators B. Chem. 81, 316-328.

- Cunningham, B., Li, P., Schulz, S., Lin, B., Baird, C., Gerstenmaier, J., Genick, C., Wang, F., Fine, E., Laing, L., 2004. J. Biomol. Screen. 9, 481-490.

- Erhardt, J.G., Estes, J.E., Pfeiffer, C.M., Biesalski, H.K., Craft, N.E., 2004. J. Nutr. 134, $3127-3132$

- Gallegos, D., Long, K., Yu, H., Clark, P., Lin, Y., George, S., Nath, P., Cunningham, B., 2013. Lab on a Chip. 13, 2124-2132.

- Heeres, J.T., Hergenrother, P.J., 2011. Chem. Soc. Rev. 40, 4398-4410.

- Horton, S., Ross, J., 2003. Food Policy. 28, 51-75.

- Jakstys, B., Klintsova, K., 2006. Available online at: https://itg.beckman.illinois.edu/microscopy_suite/equipment/downloads/TEM_instructio ns.pdf, Last Accessed: July 6th, 2015. 
- Kyprianou, D., Chianella, I., Guerreiro, A., Piletska, E.V., Piletsky, S.A., 2013. Talanta. $103,260-266$.

- $\quad$ Lee, K., Lee, M., Byun, K., Lee, I. J., 2011. Mat. Chem. 21, 5156-5162.

- $\quad$ Lu, A.H., Salabas, E.L., Schü th, F., 2007. Angew. Chem. Int. Ed. 44, 1222-1244.

- Marks, V., 2002. Clin. Chem. 48, 2008-16.

- Mathias, P.C., Jones, S.L., Wu, H., Yang, F., Ganesh, N., Gonzalez, D.O., Bollero, G., Vodkin, L.O., Cunningham, B.T., 2010. Anal. Chem. 82, 6854-6861.

- Mei, Z., Pfeiffer, C., Looker, A., Flores Ayala, R., Lacher, D., Mirel, L., Grummer Strawn, L., 2012. Clin. Chim. Acta. 413, 1479-84.

- Petersen, P. H., Stöckl, D., Blaabjerg, O., Pedersen, B., Birkemose, E., Thienpont, L., Lassen, J. F., Kjeldsen, J., 1997. Clin. Chem. 43, 2039-2046.

- Peterson, R., Cunningham, B., Andrade, J., Biosens. Bioelectron. 2014a. 56, 320-327.

- Peterson, R., Cunningham, B., Andrade, J., 2014b. FASEB J. (813.7), 28.

- Pfeiffer, C., Cook, J., Mei, Z., Cogswell, M., Looker, A., Lacher, D., 2007. Clin. Chim. Acta. $382,112-116$.

- Phiri, K.S., Calis, J.C.J., Siyasiya, A., Bates, I., Brabin, B., van Hensbroek, M.B., 2009. J. Clin. Pathol. 62, 1103-6.

- Sachdev, H., Gera, T., Nestel, P., 2005. Public Health Nutr. 8, 117-32.

- Saha, B., Evers, T., Prins, M.W., 2014. J. Anal. Chem. 86, 8158-8166.

- Sazawal, S., Black, R., Ramsan, M., Chwaya, H., Stoltzfus, R., Dutta, A., Dhingra, U., Kabole, I., Deb, S., Othman, M., Kabole, F., 2006. Lancet. 367, 133-143.

- Shafiee, E., Lidstone, M., Jahangir, F., Inci, D., Kuritzkes, Cunningham, B.T., Demirci, U., 2014. Scientific Reports. 4, 4116. 
- Soelberg, S., Stevens, R., Limaye, A., Furlong, C., 2009. Anal. Chem. 81, 2357-63.

- Speeckaert, M.M., Speeckaert, R., Delanghe, J.R., 2010. Crit. Rev. Clin. Lab. Sci. 47, $213-228$

- Thorpe, S., Heath, A., Sharp, G., Cook, J., Ellis, R., Worwood, M., 2010. Clin. Chem. Lab. Med. 48, 815-20.

- Ward, J.H., 1987. Invest. Radiol. 22, 74-83

- World Health Organization. 2008. WHO World Wide Prevalence of Anemia 1993-2005: WHO Global Database on Anemia. De Benoist, D., McLean, E., Egli, I., Cogswell, M., eds. Geneva, WHO.

- Worwood, M., 1979. CRC Crit. Rev. Clin. Lab. Sci. 10, 171-204.

- Xu, H., Aguilar, Z.P., Yang, L., Kuang, M., Duan, H., Xiong, Y., Wei, H., Wang, A., 2011. Biomaterials. 32, 9758-9765.

- Yang, J., Gunn, J., Dave, S., Zhang, M., Wang, Y.A., Gao, X., 2008. Analyst. 133, 15460. 
Table 1. Precision of various methods measuring sTfR in Liquichek ${ }^{\mathrm{TM}}$ sera.

\begin{tabular}{lccccc}
\hline Sensing Platform & $\begin{array}{c}\text { LoD } \\
(\mathrm{ng} / \mathrm{mL})\end{array}$ & $\begin{array}{c}\text { Intra-assay } \\
\% \text { RSD }\end{array}$ & $n^{1}$ & $\begin{array}{c}\text { Inter-assay } \\
\% \text { RSD }\end{array}$ & $n^{2}$ \\
\hline Standard Sandwich & 14 & 22.2 & 3 & 3.9 & 5 \\
Inverse Sandwich & 21 & 8.1 & 3 & 18.2 & 6 \\
BioVendor ELISA & 100 & 1.2 & 3 & 12.9 & 6 \\
R\&D ELISA & 553 & 2.4 & 3 & 13.2 & 5 \\
\hline
\end{tabular}

${ }^{1}$ Number of replicates in intra-assay experiments.

${ }^{2}$ Number of total replicates in two inter-assay experiments (i.e. 2-3 replicates per experiments). 
Fig. 1.

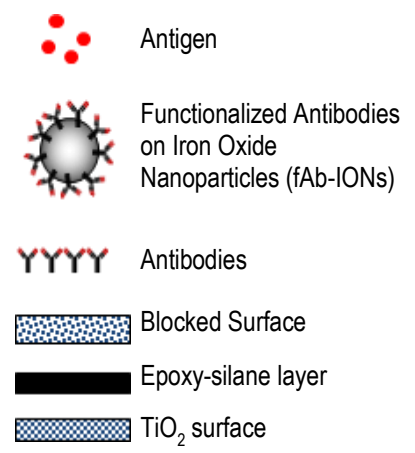

A

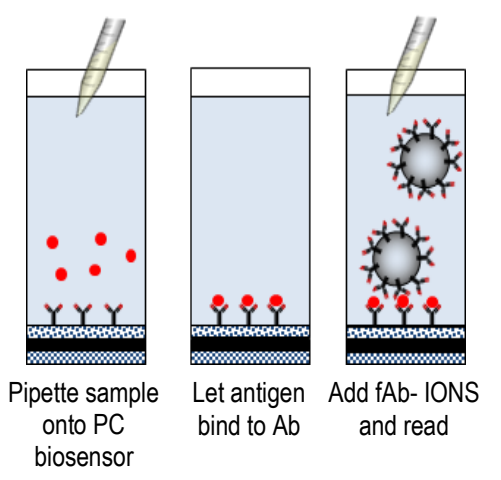

B

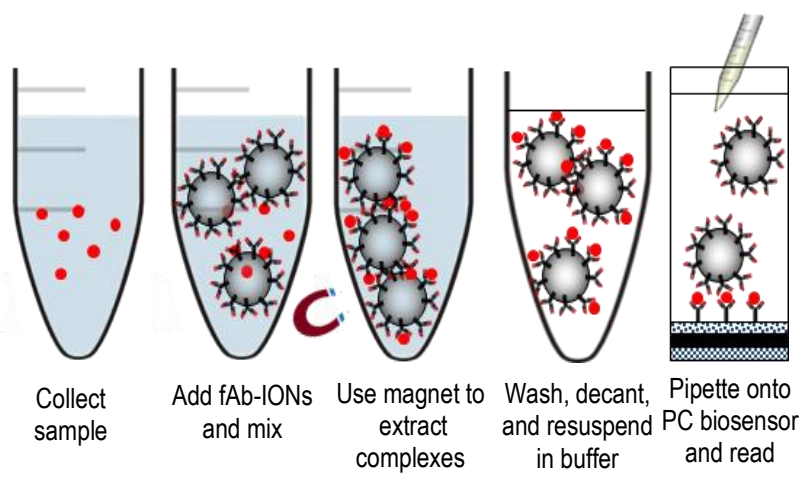


Fig. 2.

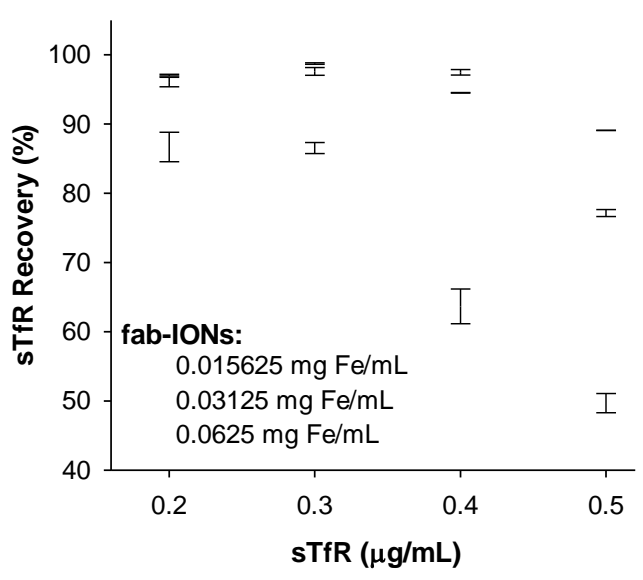


Fig. 3.
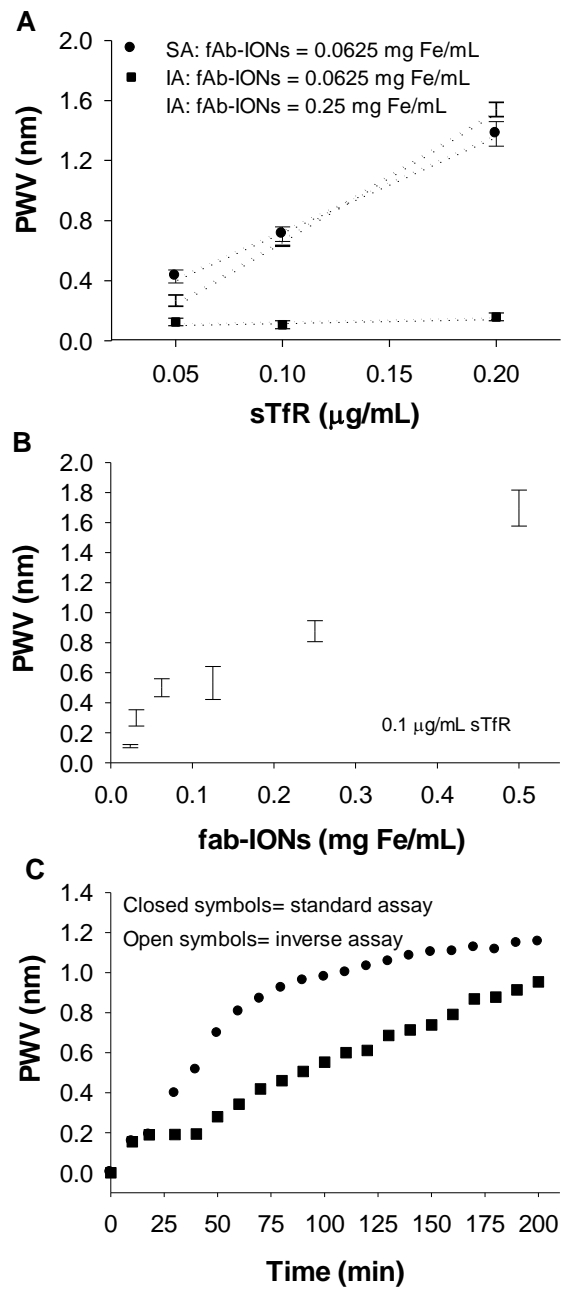
Fig. 4

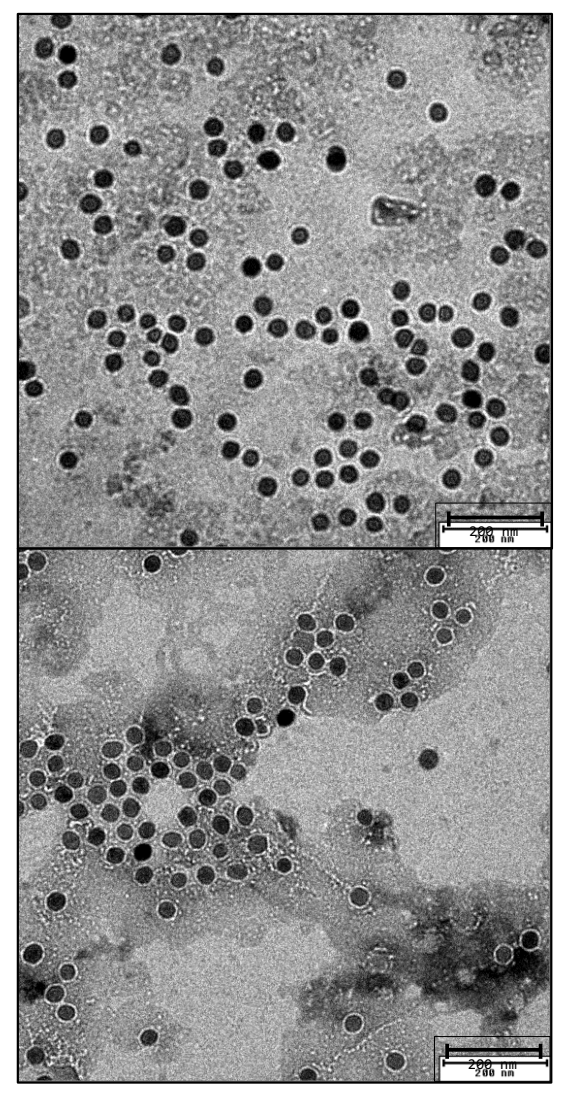


Fig. 5

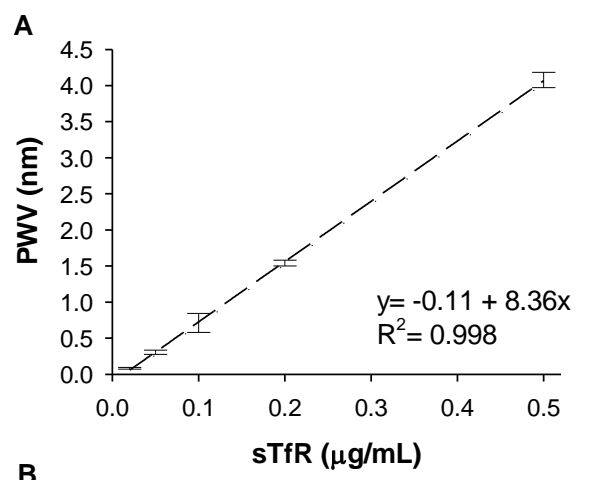

B

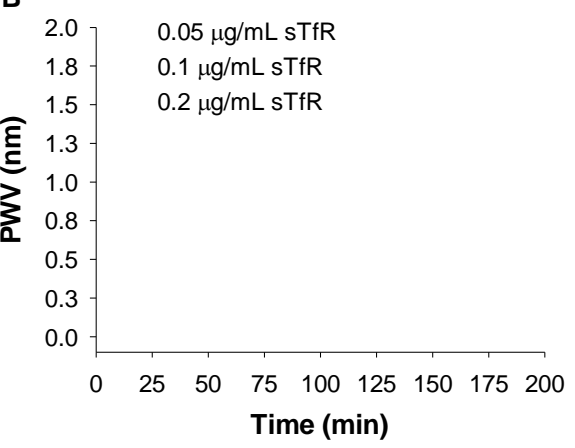


Fig. 6

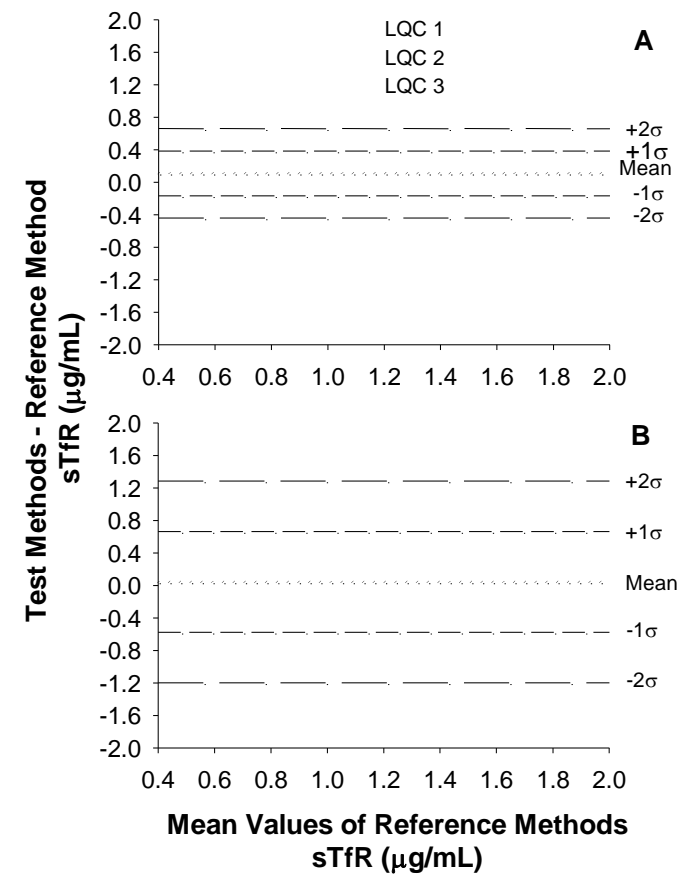




\section{Figure Captions}

Fig. 1. A) Detection steps for standard sandwich assay (SA); and B) inverse sandwich assay (IA).

Fig. 2. Recovery (\%) of different concentrations of sTfR in buffer after $1 \mathrm{~h}$ of shaking (400 rpm) at room temperature, followed by $1 \mathrm{~h}$ magnetic separation using increasing concentrations of fAb-IONs.

Fig. 3. Optimization of fAb-IONs concentration to maximize dose-response measurement of sTfR in the inverse sandwich assay (IA). A) Comparison of linear responses when using IA at 0.0625 and $0.25 \mathrm{mg}$ $\mathrm{Fe} / \mathrm{mL}$ with standard assay (SA) at $0.0625 \mathrm{mg} \mathrm{Fe} / \mathrm{mL}$ to measure sTfR on the PC biosensor. Responses were measured at $200 \mathrm{~min}$. B) PWV responses of $0.1 \mu \mathrm{g} / \mathrm{mL}$ sTfR on the IA using several fAb-ION concentrations. C) Binding kinetics of $\operatorname{sTfR}(0.1 \mu \mathrm{g} / \mathrm{mL})$ from SA and IA using fAb-ION concentrations, 0.0625 (squares) and 0.25 (circles) $\mathrm{mg} \mathrm{Fe} / \mathrm{mL}$, on PC biosensor.

Fig. 4. Transmission electron microscope images of sTfR-fAb-IONs A) before and B) after magnetic separation.

Fig. 5. Inverse sandwich assay response on PC biosensor platform. A) Standard curve using several sTfR concentrations and $0.25 \mathrm{mg}$ Fe/mL fAb-IONs. B) Representative kinetic binding curves. Data points represent means and errors bars represent SDs from triplicates.

Fig. 6. Difference plots comparing concentrations of sTfR in the IA (A) and in the SA (B) on the PC biosensor to those from commercial ELISAs. For both difference plots, three Liquichek ${ }^{\mathrm{TM}}$ control sera (LQC) were measured in either duplicate or triplicate on each platform in two experiments on different days. 